\title{
Características fisicoquímicas de nueve híbridos de sorgo (Sorghum bicolor L. Moench) y su relación con el contenido de almidón y digestión ruminal
}

\author{
Physicochemical characteristics of nine sorghum \\ (Sorghum bicolor L. Moench) hybrids and their \\ relationship to starch content and ruminal digestion
}

\author{
Mariana Villarreal-Silvaa, Francisco Castrejón Pinedaa, Alejandro Plascenciab, Lourdes \\ Carolina Pujolb, Alfredo Estrada-Anguloc, Francisco Gerardo Ríos-Rincónc, J esús Manuel \\ Cortez Sáncheza, Manuel Cuca-Garcíad, Luis Coronaa
}

\begin{abstract}
RESUMEN
Nueve híbridos de sorgo (Sorghum bicolor L. Moench) se analizaron para determinar la relación de sus características físicas [materia seca (MS), peso de mil granos (PMG), tamaño de partícula (TP), densidad relativa (DR), superficie de área (SA), partículas por gramo (PPG), endospermo duro (END-DU), endospermo blando (ENDBL)]; y químicas [nitrógeno total (NT), fibra detergente ácido (FDA), fibra detergente neutro (FDN), contenido de almidón (ALM), almidón soluble (AlmS), almidón insoluble (AlmNS). La digestión ruminal esperada (DRUM) se estimó con las fracciones de AlmS y AlmNS in vitro. El promedio de PMG, TP, DR, SA, PPG y END-DU fue de $24.61 \mathrm{~g}, 3.17 \mathrm{~mm}, 1.30 \mathrm{~g} / \mathrm{cm}^{3}, 17.44 \mathrm{~cm}^{2} / \mathrm{g}, 30.33$ y $46.68 \%$, respectivamente El promedio (\%) de MS, PC, FDN, FDA, ALM, AlmS y AlmNS fue de 92.47, 8.19, 19.17 y 8.30, 69.58, 6.00 y 47.74 respectivamente. La concentración de taninos fue baja (<0.003 eqCat). La DRUM promedió $66 \%$ (rango 64.6 a $68.4 \%$ ). No se detectó ninguna relación $(P>0.05)$ entre las variables físicas o químicas con el contenido total de almidón en el grano; sin embargo las variables densidad relativa y superficie de área, permiten incrementar el poder de predicción de la digestibilidad ruminal del almidón (DRUM, \% $=74.058-0.108 E N D-D U+0.0275 A-5.887 D R, R^{2}=0.77$, $P<0.05)$. De acuerdo a los resultados obtenidos, las variedades estudiadas son de dureza intermedia, libres de taninos y con un potencial de digestibilidad ruminal del almidón del 66 \%.
\end{abstract}

PALABRAS CLAVE: Sorgo bicolor, Almidón, Digestión, Composición fisicoquímica.

\begin{abstract}
Nine grain sorghum hybrids (bicolor Sorghum L. Moench) were analyzed to evaluate the relationship between the physical [dry matter (DM), thousand grains weigh (TGW), particle size (PS), relative density (RD), area surface (AS) and particles for gram (PG), hard endosperm (HE) and soft endosperm (SE)]; and chemical characteristics [total nitrogen (N), acid detergent fiber (ADF), neutral detergent fiber (NDF), total starch (S), soluble starch (S-S), insoluble starch (I-S)]. The predicted ruminal starch digestion (PRSD) was determined enzymatically from the S-S and I-S fractions. The average of TGW, PS, RD, AS, PG and HE were $24.61 \mathrm{~g}, 3.17 \mathrm{~mm}, 1.30 \mathrm{~g} / \mathrm{cm}^{3}, 17.44 \mathrm{~cm}^{2} / \mathrm{g}, 30.33 \mathrm{and}$ $46.68 \%$ respectively. The average (\%) of DM, CP, FDN, FDA, S, S-S and I-S were 92.47, 8.19, 19.17, 8.30 69.58, 6.00 and 47.74 respectively. The concentration of tannins was very low $(<0.003$ eqCat). The PRSD averaged $66.04 \pm$ $1.24 \%$ (range 64.61 to 68.42). No relationship was detected ( $P>0.05)$ among the physical or chemical variables with the total content of starch in the grain; however, the variables relative density and area surface, significantly increases prediction (PRSD, \% = 74.058 - 0.108HE + 0.027AS - 5.887RD, $\left.R^{2}=0.77, P<0.05\right)$. According to the study, the sorghum hybrids are of intermediate hardness, free of tannins and with a potential of ruminal starch digestion of $66 \%$.
\end{abstract}

KEY WORDS: Sorghum bicolor, Starch, Digestion, Physicochemical composition.

Recibido el 18 de agosto de 2014. Aceptado el 22 de septiembre de 2014.

a Facultad de Medicina Veterinaria y Zootecnia, Universidad Nacional Autónoma de México. Departamento de Nutrición Animal y Bioquímica. Cd. Universitaria, México, D.F. 04510. gochi@unam.mx. Correspondencia al último autor.

b Instituto de Ciencias Veterinarias, Universidad Autónoma de Baja California. México.

c Facultad de Medicina Veterinaria y Zootecnia, Universidad Autónoma de Sinaloa. México.

d Colegio de Posgraduados. Recursos Genéticos y Productividad. Montecillo, Estado de México. México. 


\section{INTRODUCCIÓN}

Como resultado de su aporte energético, el sorgo en grano (Sorghum bicolor L. Moench) es uno de los cereales más utilizados en México en la engorda de bovinos en confinamiento. La densidad energética del grano de sorgo obedece a su alto contenido de almidón (68 a $72 \%)^{(1)}$; sin embargo, la estructura y composición de los almidones y su interacción con las proteínas determinan la digestibilidad del almidón contenido en el grano y en el valor energético disponible del mismo(2). El desarrollo de híbridos en el sorgo se ha dirigido principalmente a obtener mayores rendimientos de grano, menor uso de agua, incremento en la resistencia a plagas, así como el aumento o disminución en la concentración de ciertos compuestos de importancia para la industria(3). La concentración de almidón es la menos afectada durante el desarrollo de los híbridos (4), pero como resultado de las condiciones donde los híbridos son producidos (altas temperaturas y poca agua), comparado con el maíz, las digestibilidades in vitro e in vivo del almidón de los híbridos del grano de sorgo han demostrado ser más variables $(5,6)$. Lo anterior se refleja en las diferencias observadas en el comportamiento productivo del ganado alimentado con diferentes híbridos de sorgo(7). Está demostrado que el desarrollo de nuevos híbridos afecta en forma diferente las relaciones de las principales características fisicoquímicas (principalmente proteína y proporción de endospermo duro:blando) y por lo tanto, esto puede modificar la digestibilidad de sus componentes $(8,9)$. Aunque recientemente se ha sugerido que la proporción de endospermo duro:blando es uno de los factores más importantes que limita la digestión del almidón del grano de maíz en rumiantes(10), existe información limitada sobre las relaciones de los componentes fisicoquímicos de híbridos de sorgo con la proporción de endospermo y con la digestibilidad ruminal del almidón(11).

El objetivo del presente estudio fue describir las principales características fisicoquímicas, la

\section{INTRODUCTION}

Due to its high energy content, sorghum (Sorghum bicolor L. Moench) is one of the most widely used cereals in Mexico for feedlot cattle. This grain's energy density comes largely from its high starch content (68 to $72 \%)^{(1)}$. Grain starch content digestibility and its available energy value are influenced by starch structure and composition, and its interaction with proteins(2). Development of sorghum hybrids has focused mainly on increasing grain yields; lowering water use; augmenting pest resistance; and increasing or decreasing the concentrations of specific compounds important in industrial applications. Starch concentration is affected only minimally during hybrid development(4). However, the conditions under which hybrids are grown (e.g. high temperatures, low water availability) can cause notable variability in the in vitro and in vivo digestibilities of starch from hybrid sorghum grain $(5,6)$. Differences in digestibilities can cause differences in the productive performance of cattle fed different types of hybrid sorghum(7). New sorghum hybrids have different ratios between the principal grain physicochemical characteristics (mainly protein and the hard:soft endosperm ratio), and these can modify component digestibility $(8,9)$. Recent research using corn grain suggests the hard:soft endosperm ratio is one of the most important factors limiting digestion of starch in ruminants(10). Despite its importance, little data has been generated on the relationships between the physicochemical components of hybrid sorghum grain, endosperm proportions and ruminal starch digestion(11).

The objectives of the present study were to describe the main physicochemical characteristics and the hard:soft endosperm ratio of nine sorghum hybrids grown in Mexico, and to analyze the relationship of these parameters to starch digestibility. 
HÍBRIDOS DE SORGO Y SU RELACIÓN CON EL CONTENIDO DE ALMIDÓN Y DIGESTIÓN RUMINAL

proporción de endospermo duro y blando y su relación con la digestibilidad del almidón de nueve híbridos de sorgo cultivados en México.

\section{MATERIALES Y MÉTODOS}

Híbridos utilizados

Nueve muestras de híbridos comerciales de grano de sorgo fueron cultivadas y cosechadas en el año 2004 en el estado de Morelos para evaluar sus características fisicoquímicas y sus relaciones con la digestibilidad del almidón. Los híbridos utilizados fueron: 1) Pioneer 8282 (P8282), 2) Pioneer 8418 (P-8418), 3) DeKalb 2000 (DK-2000), 4) Asgrow Kilate (Kilate), 5) Asgrow Ámbar (Ámbar), 6) Asgrow Marfil (Marfil), 7) WAC-685, 8) Sorghum Partners Inc. KS955 (KS955) y 9) Dekalb D-65 (D-65).

Procesamiento de muestras y análisis de laboratorio

Las muestras se procesaron en el Laboratorio de Bromatología del Departamento de Nutrición Animal y Bioquímica de la Facultad de Medicina Veterinaria y Zootecnia de la Universidad Nacional Autónoma de México (UNAM). A las muestras se les retiró manualmente los materiales extraños y se conservaron en refrigeración ( $4 \stackrel{\circ}{ }$ C) hasta su procesamiento y análisis.

De cada híbrido se obtuvo una muestra contractual utilizando el método de cuarteo, y para los análisis físicos, una parte de ésta se dejó como grano entero, el resto se molió para someterla a análisis químicos.

Análisis físicos. Se realizaron por duplicado las siguientes determinaciones para el grano entero: 1) materia seca $(\mathrm{MS})^{12}$; 2) tamaño de partícula (TP, expresada como diámetro medio geométrico), para lo cual se utilizaron seis diferentes cribas: USBS \#4 $(4,760 \mu \mathrm{m})$, USBS \#5 $(4,000 \mu \mathrm{m})$, USBS \#6 $(3,360 \mu \mathrm{m})$, USBS \#7 $(2,830 \mu \mathrm{m})$, USBS \#8 $(2,380 \mu \mathrm{m})$ y USBS \#10 $(2,000 \mu \mathrm{m})$; que fueron sobrepuestas, de mayor a menor abertura en un agitador de cribas (W.S.

\section{MATERIALS AND METHODS}

Sorghum hybrids

Analyses of physicochemical characteristics and their relationship to starch digestibility were done using nine commercial sorghum hybrids harvested in 2004 in the state of Morelos, Mexico: Pioneer 8282 (P-8282); Pioneer 8418 (P-8418); DeKalb 2000 (DK-2000); Asgrow Kilate (Kilate); Asgrow Ámbar (Ámbar); Asgrow Marfil (Marfil); WAC-685; Sorghum Partners Inc. KS955 (KS955); and Dekalb D-65 (D-65).

Sample processing and laboratory analyses

Samples were processed at the Food Science Laboratory of the Animal Nutrition and Biochemistry Department of the Faculty of Veterinary Science and Zootechny, National Autonomous University of Mexico (Universidad Nacional Autónoma de México - UNAM). Samples were cleaned of all foreign material and stored in refrigeration (4 o C) until processing and analysis.

The quarter method was used to take a representative sample of each hybrid. One portion was left as whole grain for the physical analyses, while the rest was ground for chemical analyses.

Physical analyses. Duplicate analyses were done of the whole grain to quantify four parameters: 1) Dry matter content (DM)(12); 2) Particle size (PS) ${ }^{(13)}$; 3) Thousand-grain weight (TGW); and 4) Relative density (RD). Following Baker and Hermann(13), particle size was expressed as geometric mean diameter, and the grains separated using a screen shaker (W.S. Tyler, Model RX-812; Mentor, OH, USA) equipped with six US standard (National Institute of Standards and Technology - NIST) mesh sizes: \#4 (4,750 $\mu \mathrm{m})$; \#5 (4,000 $\mu \mathrm{m}) ; \quad \# 6(3,350 \mu \mathrm{m}) ; \quad$ 7 $(2,800 \mu \mathrm{m}) ; \# 8(2,360 \mu \mathrm{m}) ;$ and \#10 $(2,000$ $\mu \mathrm{m})$. Relative density was quantified based on water displacement and measured with a volumetric pycnometer.

Chemical analyses. Samples were ground and then screened through \#10 $(2,000 \mu \mathrm{m})$ mesh. 
Tyler Modelo RX-812; Mentor, OH, USA) siguiendo el procedimiento descrito por Baker y Hermann (13); 3) peso de 1,000 granos (PMG); 4) densidad relativa (DR), determinada por desplazamiento de agua utilizando picnómetro volumétrico.

Análisis químicos. Las muestras se molieron y tamizaron con criba United States Bureau of Standard (USBS) \#10 (1,680 micrones) para realizar los siguientes análisis: materia seca (MS), nitrógeno total (N) 12 , fibra detergente neutro (FDN) y fibra detergente ácido (FDA) 14 . Una segunda submuestra de cada híbrido fue molida y tamizada con criba USBS \#40 (420 micrones) para la determinación de taninos (TAN)(15) y almidón(16).

La solubilidad del almidón se determinó mediante una prueba enzimática in vitro incubándose las muestras a $39{ }^{\circ} \mathrm{C}$ durante $4 \mathrm{~h}$ para determinar la fracción de almidón soluble (AlmS) y durante $6 \mathrm{~h}$ para el almidón insoluble (AlmNS) siguiendo la técnica descrita por Rodríguez et al(17). Para preparar la solución buffer se utilizaron las enzimas amiloglucosidasa de Aspergillus niger (Sigma A-7420), $\alpha$-amilasa de páncreas porcino (Sigma 10080), pancreatina porcina (Sigma P-1625), achromopeptidasa de Achromobacter lyticus (Sigma A-3547), y proteasa de páncreas bovino (Sigma P-4630).

\section{Cálculos}

Para la estimación de superficie de área (SA) y número de partículas por grano (PPG) se utilizaron las fórmulas informadas por Baker y Herrman(13) utilizando para ello los datos de distribución de partícula. Para calcular el valor de la digestibilidad ruminal de almidón esperada (DRUM) se calcularon primero los valores de almidón reactivo a la amiloglucosidasa (AGR), almidón reactivo (RS), almidón reactivo insoluble (IRS) y almidón digerible insoluble (ISD) mediante los valores observados para el contenido de almidón del grano, así como los valores AlmS y AlmNS determinados en la prueba de digestión in vitro mediante las
Analyses were done to measure dry matter (DM), total nitrogen (TN)(12), neutral detergent fiber (NDF), and acid detergent fiber (ADF) (14). A subsample from each hybrid was ground and screened through US standard mesh \#40 (425 $\mu \mathrm{m})$ mesh to allow measurement of tannins (TAN) (15) and starch (ST) content(16).

Starch solubility was measured by an in vitro enzymatic test (17). Starch solutions were heated to $39{ }^{\circ} \mathrm{C}$ for $4 \mathrm{~h}$ to quantify the soluble starch (SST) fraction and for $6 \mathrm{~h}$ to quantify the insoluble starch (IST) fraction. The buffer solution was prepared using amyloglucosidase enzymes from Aspergillus niger (Sigma A-7420), $\alpha$-amylase from pig pancreas (Sigma 10080), pig pancreatin (Sigma P-1625), achromopeptidase from Achromobacter lyticus (Sigma A-3547), and protease from bovine pancreas (Sigma P-4630).

\section{Calculations}

Surface area (SA) and number of particles per grain (PPG) were calculated with formulas based on Baker and Herrman(13), and using particle distribution data. Predicted ruminal starch digestion (RSD) was determined by first calculating the values for starch reactive to amyloglucosidase (SRA), reactive starch (RS), insoluble reactive starch (IRS) and insoluble digestible starch (IDS). These were calculated using grain starch content (ST), SST and IST values with these formulas:

$\mathrm{SRA}=(\mathrm{SST} / \mathrm{ST}) * 100 ; \mathrm{RS}=(\mathrm{IST} / \mathrm{ST}) * 100 ; \mathrm{IRS}=$ $(R S-S R A) / 6 ;$ IDS $=(100-S R A) *(I R S /(I R S+0.05))$

Where 0.5 is the grain rumen transit rate. The RSD was then calculated as follows(17):

RSD, $\%=(1.32 * \mathrm{SRA})+(0.93 *$ IDS $), \mathrm{R}^{2}=$ 0.78

Hemicellulose (HEMI) concentration was calculated by the arithmetic mean of NDF ADF for each hybrid. Proportions of hard (HE) and soft endosperm (SE) were estimated by quantifying and differentiating their areas in the grain. For this purpose, 20 whole grains per 
siguientes fórmulas:

$\mathrm{AGR}=(\mathrm{AlmS} / \mathrm{ALM}) * 100 ; \mathrm{RS}=(\mathrm{AlmNS} / \mathrm{ALM}) *$ $100 ; \quad I R S=(R S-A G R) / 6 ; \quad I S D=(100-$ $A G R) *(I R S /(I R S+0.05))$

Donde el 0.05 representa la tasa de pasaje de grano en el rumen. La DRUM se calculó utilizando la siguiente ecuación(17):

DRUM, \% $=(1.32 *$ AGR $)+(0.93 *$ ISD $)$, $\mathrm{R}^{2}=0.78$

La concentración de la hemicelulosa (HEMICEL) se determinó mediante la diferencia aritmética de la media de FND - FDA de cada híbrido. La estimación de las proporciones de endospermo duro (END-DU) y endospermo blando (END$\mathrm{BL})$ se realizó mediante cuantificación y diferenciación de sus áreas en el grano. Para ello, de 50 granos limpios y procesados se seleccionaron a conveniencia 20 granos enteros de cada híbrido y la estimación se realizó mediante una modificación de la técnica de Kirleis et al(18) que se describe a continuación: se prepararon portaobjetos cubriendo la superficie con resina epóxica conocida como poliéster (Fusión Polymers S.A. de C.V., México), inmediatamente se colocaron sobre ésta 10 granos de sorgo de un mismo híbrido por portaobjetos, dejando suficiente espacio entre ellos hasta montar 50 granos, de tal forma que la mitad de cada grano sobresalía del poliéster y el resto se encontraba sumergido en éste. Los portaobjetos se dejaron polimerizar durante $24 \mathrm{~h}$ a temperatura ambiente ( $\left.\sim 25^{\circ} \mathrm{C}\right)$. Posteriormente, cada grano se seccionó a la mitad con un bisturí (mango Totmedical No. 3, hoja No. 22) retirando la parte que sobresalía del poliéster, exponiendo así el germen y las áreas de END-DU y END-BL. Considerando la integridad de la superficie de corte, la regularidad de los bordes del grano, la exposición completa del centro del germen y el estado sano de cada grano se seleccionaron los 20 granos de cada híbrido, sin tomar en consideración las diferencias de color, tamaño o forma. Los cortes se conservaron en sus portaobjetos en refrigeración ( $4 \stackrel{\circ}{\circ}$ ) hasta la toma de fotografías digitales. hybrid were randomly chosen from a lot of 50 clean, processed grains. Estimation was done using a modification of the technique of Kirleis et al(18). Briefly, slides were covered with epoxy resin polyester (Fusión Polymers S.A. de C.V., Mexico), and ten grains of the same hybrid placed on each slide, ensuring that half the grain remained above the polyester. Fifty grains per hybrid were mounted (i.e. five slides per hybrid). After polymerization for $24 \mathrm{~h}$ at $\sim 25 \stackrel{\circ}{ } \mathrm{C}$, a scalpel (Totmedical, No. 3 blade, No. 22 blade) was used to cut off the half of each grain above the polyester. This exposed the germ, the HE and the SE. Based on cut surface integrity, grain edge regularity, complete exposure of grain center and grain health, 20 grains per hybrid were chosen; grain color, size and shape were not taken into consideration. Cut grains were kept in the slides, under refrigeration ( $4 \stackrel{\circ}{\circ}$ ) until digital images were taken.

Each of the 20 grains from each hybrid were photographed at 7.1 MP resolution using a digital camera (PowerShot S70, Canon, Lake Success, NY) and a stereoscopic microscope (Leica Z125) with a camera adapter (Leica DS150). Images were focused manually, no flash was used and contrast was set at "exterior". I mages were edited using Adobe Photoshop Elements(19). The area of the germ $\mathrm{HE}$, the pericarp and area of the germ SE, and the HE were marked with a black line ( $0 \%$ color saturation; blue, green and red tones at $0 \%$; $0 \%$ brightness, \#:000000; Hard round 5 pixels). The lines must exhibit no interruptions or flaws since this can effect later selection and measuring of areas in pixels. The areas of the HE and SE were calculated with the "Magic Wand Tool", and the number of pixels in this area was treated as total endosperm area. The number of pixels accounting for $\mathrm{HE}$ was calculated by substraction, and the percentages of $\mathrm{HE}$ and $\mathrm{SE}$ within the total endosperm area were calculated.

\section{Statistical analysis}

To calculate differences between hybrids in terms of the hard:soft endosperm percentages, each image was established as an experimental unit. 
De cada uno de los 20 granos seleccionados por cada híbrido se tomaron fotografías con una cámara Canon PowerShot S70 (7.1 MP, Lake Success, NY) y un microscopio estereoscópico Leica Z125 con adaptador de cámara Leica DS150, enfocando manualmente cada toma, sin flash y ajustando el contraste de blancos al modo "exterior". Cada fotografía se editó utilizando el software Adobe Photoshop Elements(19). Se delimitó con color negro (saturación de color $0 \%$, tonos: azul verde y rojo al $0 \%$, brillo $0 \%$, \#:000000) y grosor de línea de 5 píxeles (Hard round 5 pixels) el área de END-DU del germen, el pericarpio y el área de END-BL del germen y el END-DU con el uso de la función "Pencil tool" seleccionada en la barra de "Tools"; las líneas trazadas no debían presentar ninguna interrupción o corte, ya que esto afecta la selección de las áreas y su medición posterior en píxeles. Se determinó el área de END-DR y END-BL utilizando la función "Magic Wand Tool". Se revisó de nuevo el número de píxeles que se encontraron dentro de esta área, y así se obtuvo el número de píxeles dentro del endospermo total. Por medio de sustracción aritmética se obtuvo el número de píxeles del END-DR y se calculó la proporción porcentual de END-DR y END-BL respecto al total de endospermo.

\section{Análisis estadístico}

Para calcular la diferencia entre los híbridos en la proporción de endospermo duro:blando se estableció como unidad experimental cada fotografía que cumpliera con las características mencionadas. Los datos obtenidos de la proporción de endospermo duro:blando se analizaron mediante el Procedimiento de Modelos General Linear (GLM, SAS)(20) y las diferencias entre medias se evaluaron mediante la Prueba de Tukey con valor de significancia de $P<0.05(21)$. Se realizaron correlaciones simples ( $r$ de Pearson) entre todas las características físicoquímicas y se utilizó el Modelo de Regresión Lineal Simple para las correlaciones de: END-DR y END-BL, FAD y CEL, FAD y $\mathrm{N}$, PMG y TP, SA y DRA y DRA y END-DR y se
The hard:soft endosperm percentage data were analyzed using the General Linear Models (GLM) procedure in the SAS program(20). Differences between the means were evaluated with a Tukey test (significance $P<0.05)(21)$. Simple (Pearson $r$ ) correlations were run between all the physicochemical characteristics, and a simple linear regression model used for the correlations between HE and SE; ADF and CEL; ADF and TN; TGW and PS; SA and RSD; and RSD and $\mathrm{HE}$. A multiple regression equation was run to predict RSD using the $H E, S A$ and RD variables with the Stepwise Linear Regression function in the Statistix program(22).

\section{RESULTS}

Physical characteristics

Particle size (PS), TGW and DR were highest in the Kilate hybrid. The lowest TGW was recorded in the P-8282 hybrid, and the lowest RD values $(<1.290)$ were in the Marfil, Ambar and DK2000 hybrids (Table 1). Data for average PS, SA and PPG showed that more than $97 \%$ of particles were within the 2.83 to $3.99 \mathrm{~mm}$ range (Table 2). Within this range, $62 \%$ (Kilate) to

Cuadro 1. Características físicas del grano de nueve híbridos de sorgo

Table 1. Grain physical characteristics of nine sorghum hybrids

\begin{tabular}{lcccc}
\hline Hybrid & $\begin{array}{c}\text { TGWa } \\
(\mathrm{g})\end{array}$ & $\begin{array}{c}\mathrm{DM}^{\mathrm{a}} \\
(\%)\end{array}$ & $\begin{array}{c}\text { PSa } \\
(\mu \mathrm{m})\end{array}$ & $\begin{array}{c}\mathrm{RD}^{\mathrm{a}} \\
\mathrm{g}^{\mathrm{cm}} \mathrm{cm}^{3}\end{array}$ \\
\hline P-8282 & 22.37 & 92.43 & 3145 & 1.298 \\
DK-2000 & 24.05 & 92.09 & 3137 & 1.263 \\
Kilate & 27.75 & 93.16 & 3290 & 1.309 \\
D-65 & 24.20 & 92.45 & 3161 & 1.308 \\
WAC-685 & 24.38 & 92.81 & 3167 & 1.317 \\
Ambar & 25.98 & 91.65 & 3193 & 1.275 \\
P-8418 & 23.88 & 92.31 & 3147 & 1.323 \\
KS-955 & 25.29 & 92.21 & 3168 & 1.307 \\
Marfil & 23.55 & 93.13 & 3100 & 1.286 \\
Average & 24.61 & 92.47 & 3168 & 1.299 \\
Standard deviation & 1.56 & 0.49 & 52.53 & 0.02 \\
\hline
\end{tabular}

aAverage by duplicate; $\mathrm{TGW}=$ thousand-grain weight; $\mathrm{DM}=$ dry matter, $\mathrm{PS}=$ particle size (geometric mean diameter); $\mathrm{RD}=$ relative density. 
HÍBRIDOS DE SORGO Y SU RELACIÓN CON EL CONTENIDO DE ALMIDÓN Y DIGESTIÓN RUMINAL

Cuadro 2. Tamaño de partícula del grano de híbridos de sorgo (mm)

Table 2. Grain particle size of nine sorghum hybrids $(\mathrm{mm})$

\begin{tabular}{|c|c|c|c|c|c|c|c|c|c|}
\hline & \multicolumn{9}{|c|}{ Hybrids } \\
\hline & \multicolumn{9}{|c|}{$\%$ whole sorghum grain } \\
\hline$>4.76$ & 0.00 & 0.00 & 0.00 & 0.00 & 0.00 & 0.00 & 0.00 & 0.00 & 0.00 \\
\hline $4.75-4.00$ & 0.03 & 0.00 & 0.00 & 0.04 & 0.00 & 0.03 & 0.08 & 0.02 & 0.00 \\
\hline $3.99-3.36$ & 13.80 & 7.35 & 22.81 & 37.99 & 17.56 & 29.03 & 18.49 & 14.61 & 12.88 \\
\hline $3.35-2.83$ & 84.55 & 90.06 & 75.50 & 61.59 & 81.23 & 70.41 & 79.19 & 85.18 & 86.48 \\
\hline $2.82-2.38$ & 1.30 & 2.03 & 1.35 & 0.31 & 0.83 & 0.35 & 1.65 & 0.13 & 0.46 \\
\hline $2.37-2.00$ & 0.28 & 0.47 & 0.27 & 0.06 & 0.33 & 0.16 & 0.56 & 0.05 & 0.16 \\
\hline$<2.00$ & 0.04 & 0.09 & 0.06 & 0.01 & 0.04 & 0.02 & 0.03 & 0.01 & 0.02 \\
\hline Total & 100.00 & 100.00 & 100.00 & 100.00 & 100.00 & 100.00 & 100.00 & 100.00 & 100.00 \\
\hline $\mathrm{PS}, \mu \mathrm{m}$ & 3145 & 3100 & 3193 & 3290 & 3168 & 3237 & 3167 & 3161 & 3147 \\
\hline $\mathrm{SA}, \mathrm{cm}^{2} / \mathrm{g}$ & 23 & 17 & 23 & 15 & 16 & 16 & 16 & 16 & 15 \\
\hline PPG & 53 & 26 & 50 & 22 & 25 & 23 & 25 & 24 & 25 \\
\hline GSD & 1.51 & 1.09 & 1.51 & 1.09 & 1.09 & 1.09 & 1.09 & 1.07 & 1.07 \\
\hline
\end{tabular}

$\mathrm{PS}=$ particle size, expressed as geometric mean diameter; $\mathrm{SA}=$ Surface area; $\mathrm{PPG}=$ particles per grain; $\mathrm{GSD}=$ geometric standard deviation.

desarrolló una ecuación de regresión múltiple para predecir DRA utilizando las variables: ENDDR, SA y DR mediante el uso de la función Stepwise Linear Regression utilizando el programa Statistix(22).

\section{RESULTADOS}

Características físicas del grano de sorgo

Las características físicas de los híbridos estudiados se muestran en los Cuadros 1 y 2 . Los valores representan el promedio del análisis por duplicado y se expresan en base húmeda. La MS, PMG, TP y densidad relativa (DR) promediaron $92.47 \pm 0.49 \%, 24.61 \pm 1.56$ $\mathrm{g}, 3,168 \pm 53 \mu \mathrm{m}, 1.299 \pm 1.02 \mathrm{~g} / \mathrm{cm}^{3}$, respectivamente. El híbrido Kilate presentó mayor PMG, TP y DR, mientras que el híbrido P-8282 obtuvo los valores más bajos para PMG. Los híbridos Marfil, Ámbar y DK-2000 registraron los valores más bajos $(<1.290)$ para DR.

La distribución del tamaño promedio de partícula, superficie de área y partículas por grano calculadas se muestran en el Cuadro 2. Más del $97 \%$ de las partículas se distribuyeron en el rango de 2.83 a $3.99 \mathrm{~mm}$ dentro de las
$90 \%$ (Marfil) fell within the 2.83 to 3.35 range. Average SA (15 to $23 \mathrm{~cm}^{2} / \mathrm{g}$ ) and PPG (22 to 53) data showed Kilate to have the lowest PPG and SA while P-8282 had the highest values.

Chemical characteristics

Average TN content among all the hybrids was $1.31 \pm 0.07 \%$, equivalent to $8.19 \%$ of the cell wall (Table 3). The highest TN was in Marfil $(1.39 \%=8.7 \%$ PC) and the lowest in Kilate $(1.15 \%=7.2 \%$ PC).

Average NDF was $19.17 \pm 2.07 \%$, and average ADF was $8.30 \pm 0.49 \%$. The hybrid D65 had a NDF content $(21.80 \%)$ that was $49 \%$ higher than the lowest NDF content in DK-2000 (14.65\%). Acid detergent fiber (ADF) contents were very similar between the hybrids; even so, PK-8418 had the highest value $(8.93 \%)$ and Kilate the lowest $(7.51 \%)$.

Cell wall components averaged $3.63 \pm 0.41 \%$ for CEL, $10.87 \pm 2.08 \%$ for HEMI and $4.54 \pm$ $0.31 \%$ for LIG. No differences were observed between hybrids in CEL, HEMI and LIG values, with one exception: HEMI in DK-2000 (6.69\%) 
Mariana Villarreal-Silva, et al. / Rev Mex Cienc Pecu 2015;6(3):243-261

Cuadro 3. Características químicas, fracciones del almidón y digestibilidad ruminal del grano de híbridos de sorgo (\%)a Table 3. Grain chemical characteristics, starch fractions and ruminal digestibility for nine sorghum hybrids (\%)a

\begin{tabular}{lccccccccccc}
\hline Hybrid & TN & NDF & ADF & CEL & HEMI & LIG & TAN (eqCat) & ST & SST & IST & RSD \\
\hline P-8282 & 1.32 & 19.03 & 8.62 & 3.94 & 10.41 & 4.52 & 0.0025 & 68.19 & 5.98 & 52.03 & 68.42 \\
DK-2000 & 1.29 & 14.65 & 7.69 & 3.02 & 6.96 & 4.48 & 0.0015 & 68.64 & 6.06 & 47.67 & 66.31 \\
Kilate & 1.15 & 20.08 & 7.51 & 3.32 & 12.57 & 4.12 & 0.0005 & 70.82 & 5.75 & 47.08 & 65.38 \\
D-65 & 1.25 & 21.80 & 8.30 & 3.91 & 13.50 & 4.28 & 0.0012 & 71.78 & 5.65 & 46.07 & 64.31 \\
WAC-685 & 1.36 & 19.59 & 8.17 & 3.49 & 11.43 & 4.51 & 0.0000 & 67.52 & 5.70 & 44.74 & 65.32 \\
Ambar & 1.32 & 20.02 & 8.09 & 3.12 & 11.93 & 4.94 & 0.0000 & 70.27 & 7.19 & 49.85 & 67.14 \\
P-8418 & 1.36 & 18.39 & 8.93 & 3.98 & 9.46 & 4.77 & 0.0000 & 70.65 & 5.83 & 46.57 & 65.00 \\
KS-955 & 1.36 & 20.97 & 8.45 & 4.14 & 12.51 & 4.24 & 0.0000 & 69.68 & 5.64 & 48.15 & 65.98 \\
Marfil & 1.39 & 17.95 & 8.90 & 3.72 & 9.06 & 5.00 & 0.0000 & 68.71 & 6.22 & 47.47 & 66.55 \\
Average & 1.31 & 19.17 & 8.30 & 3.63 & 10.87 & 4.54 & 0.00 & 69.58 & 6.00 & 47.74 & 66.04 \\
SD & 0.07 & 2.07 & 0.49 & 0.41 & 2.08 & 0.31 & 0.00 & 1.41 & 0.49 & 2.12 & 1.24 \\
\hline
\end{tabular}

a Values reported in dry base.

$\mathrm{TN}=$ total nitrogen; $\mathrm{NDF}=$ neutral detergent fiber; $\mathrm{ADF}=$ acid detergent fiber; $\mathrm{CEL}=$ cellulose; $\mathrm{HEMI}=$ hemicellulose; LIG= lignin; $T A N=$ tannins, expressed as catechin equivalents; $\mathrm{ST}=$ starch; $\mathrm{SST}=$ soluble starch; IST= insoluble starch; RSD= predicted ruminal starch digestion.

$\mathrm{SD}=$ standard deviation.

cuales del 62 al $90 \%$ fueron partículas en el rango de 2.83 a $3.35 \mathrm{~mm}$, siendo el menor $(61.59 \%)$ para el híbrido Kilate y mayor (90.06\%) para el híbrido Marfil. Los valores calculados de superficie de área (SA) y número de partículas por grano (PPG) promediaron 22-53 y 15-23 $\mathrm{cm}^{2} / \mathrm{g}$, respectivamente. El híbrido Kilate presentó una menor PPG y SA mientras que el híbrido P-8282 obtuvo los valores más altos para PPG y SA.

Características químicas del grano de sorgo El contenido de nitrógeno (NT), composición de paredes celulares y concentración de taninos se muestra en el Cuadro 3. El promedio de contenido de NT fue $1.31 \pm 0.07 \%$ el cual equivale a $8.19 \%$ de PC. El mayor contenido de NT fue para el híbrido Marfil $(1.39 \%=$ $8.7 \%$ PC) y el menor fue para Kilate $(1.15 \%$ $=7.2 \%$ PC). El promedio de FDN y FDA fue de $19.17 \pm 2.07$ y $8.30 \pm 0.49 \%$, respectivamente. El híbrido D65 presentó $49 \%$ más de FDN que DK-2000 (21.80 vs $14.65 \%$ ); por otra parte, el contenido de FDA fue muy similar entre los híbridos, aún así PK-8418 mostró el valor más alto $(8.93 \%)$ y Kilate el was notably lower than the $13.36 \%$ average among the remaining hybrids.

Tannins (TAN) were detected in only four of the nine hybrids, and at very low levels $(0.0005-0.001 \%$, expressed in catechin equivalents). These levels are so low that all nine hybrids can be considered to be essentially tannin-free.

Average starch (ST) content was $69.58 \pm$ $1.41 \%$, the lowest level $(67.52 \%)$ being in D65 and the highest $(71.78 \%)$ in WAC-685 (Table 3). The soluble starch (SST) fraction averaged $6.00 \pm 0.49 \%$ while the insoluble starch (IST) fraction averaged $47.74 \pm 2.14 \%$. Average RSD was $66.04 \pm 1.24 \%$, with the highest level $(68.42 \%)$ in P-8282 and the lowest $(64.61 \%)$ in D-65.

The HE proportion averaged $47.68 \pm 2.98 \%$ and the SE $52.32 \pm 2.98 \%$ (Table 4). The HE:SE proportion (44.63:55.37) of DK-2000, Ámbar and KS-955 differed $(P<0.05)$ from that of WAC-685 (52.24:47.76). The remaining five hybrids did not differ. 
HÍBRIDOS DE SORGO Y SU RELACIÓN CON EL CONTENIDO DE ALMIDÓN Y DIGESTIÓN RUMINAL

más bajo (7.51 \%) para FDA. La concentración de los componentes de las paredes celulares fue de $3.63 \pm 0.41 \%$ para CEL, $10.87 \pm 2.08$ $\%$ para HEMICEL y de $4.54 \pm 0.31 \%$ para LIG. Con excepción del híbrido DK-2000 el cual registró la mitad de contenido de hemicelulosa (6.96 vs $13.36 \%$ en promedio para el resto de los híbridos), los valores observados para CEL, HEMICEL Y LIG fueron similares para todos los híbridos. Sólo se detectó presencia de taninos en 4 de los 9 híbridos estudiados y los valores obtenidos fueron muy bajos (rango de 0.0005 a $0.001 \%$, expresados en equivalentes de catequina) por lo que se considera que los nueve híbridos no contenían taninos.

En contenido de almidón ( $A L M)$, las concentraciones de las fracciones soluble e insoluble de almidón así como la digestibilidad ruminal estimada se muestran en el Cuadro 3. El contenido de ALM promedió $69.58 \pm 1.41 \%$ (rango 67.52 a 71.78) siendo el valor más alto para el híbrido D65 y más bajo para el híbrido WAC-685. La fracción de almidón soluble (ALMSOL) promedió $6.00 \pm 0.49 \%$, mientras que el promedio de ALM-INSOL fue de $47.74 \pm$ $2.14 \%$.

La digestibilidad ruminal estimada (DRUM) promedió $66.04 \pm 1.24 \%$ (rango 64.61 a 68.42); resultando el valor más alto para el híbrido P82-82 y el más bajo para el híbrido D-65. La comparación en la proporción de endospermo duro:blando de los híbridos estudiados se muestran en el Cuadro 4. El promedio fue de $47.68 \pm 2.98$ y $52.32 \pm$ $2.98 \%$ para END-DU y END-BL respectivamente. Las proporciones de los híbridos DK-2000, Ámbar, KS-955 fueron diferentes $(P<0.05)$ de WAC-685 (44.63:55.37 vs 52.24:47.76, respectivamente). El resto de los nueve híbridos no fueron diferentes entre sí.

Correlación de las características físicas y químicas

Los coeficientes de correlación lineal encontrados entre los valores de todas las características
Cuadro 4. Proporción de END-DU y END-BL del grano de híbridos de sorgo

Table 4. Grain hard and soft endosperm proportions in nine sorghum hybrids

\begin{tabular}{lcll}
\hline Hybrid & Images $(\mathrm{n}=)$ & $\mathrm{HE}(\%)$ & $\mathrm{SE}(\%)$ \\
\hline WAC-685 & 20 & $52.24^{\mathrm{a}}$ & $47.76 \mathrm{a}$ \\
KILATE & 20 & $50.51^{\mathrm{ab}}$ & $49.49 \mathrm{ab}$ \\
D-65 & 20 & $50.80^{\mathrm{ab}}$ & $49.20 \mathrm{ab}$ \\
P-8418 & 20 & $48.65^{\mathrm{abc}}$ & $51.35 \mathrm{abc}$ \\
MARFIL & 20 & $47.12^{\mathrm{abc}}$ & $52.88 \mathrm{abc}$ \\
P-8282 & 20 & $45.99 \mathrm{bc}$ & $54.01 \mathrm{bc}$ \\
KS-955 & 20 & $44.73^{\mathrm{c}}$ & $55.27 \mathrm{c}$ \\
ÁMBAR & 20 & $44.18^{\mathrm{c}}$ & $55.82 \mathrm{c}$ \\
DK-2000 & 20 & $44.96^{\mathrm{c}}$ & $55.04 \mathrm{C}$ \\
Average & 52.32 & 47.68 & \\
SD & 2.98 & 2.98 & \\
\hline
\end{tabular}

$\mathrm{HE}=$ hard endosperm; $\mathrm{SE}=$ soft endosperm; $\mathrm{SD}=$ standard deviation.

abc Different letter superscripts in the same column indicate significant difference between the means $(P<0.0001)$.

Correlation of physical and chemical characteristics

Linear correlation coefficients among all the measured characteristics showed the highest strength $(P<0.05)$ in the simple correlations for physical characteristics to be 1) PS with TN ( $r=$ $-0.80)$ and ADF $(r=-0.70) ; 2)$ TGW with ADF $(r=-0.67)$ and PS $(r=0.88) ; 3)$ PPG with RSD $(r=0.81)$; 4) RD with HE $(r=0.69)$, SE $(r=$ -0.69) and SST $(r=0.70)$; and 5) SA with IST $(r=0.85)$, SST $(r=0.70)$ and RSD $(r=0.84)$ (Table 5). Among the chemical characteristics, the strongest correlations were 1) TN with LIG $(r=0.66)$ and ADF $(r=0.74) ; 2)$ NDF with HEMI $(r=0.97)$; 3) ADF with CEL $(r=0.75)$; and 4$)$ LIG with SST $(r=0.70)$. The ratio of SE to the IST fraction was 0.73 , and that between $\mathrm{HE}$ and the IST fraction was -0.73. A particularly high correlation value $(r=0.90)$ was observed between RSD and IST, whereas the correlation between RSD and SE and HE $(r=0.69)$ was notably lower.

Predictive equations

A number of significant $(P<0.05)$ relationships were identified between physicochemical 
Cuadro 5. Coeficientes de correlación (Pearson) entre la proporción de endospermo duro: blando y las características físicas y químicas del grano de sorgo

Table 5. Correlation coefficients (Pearson) between the hard: soft endosperm proportion and physical and chemical characteristics of hybrid sorghum grain

\begin{tabular}{|c|c|c|c|c|c|c|c|c|c|c|c|c|c|c|c|c|c|c|}
\hline & ST & CEL & HE & SE & ADF & NDF & HEM & LIG & DM & $\mathrm{TN}$ & TGW & RSD & SST & IST & TAN & PS & SA & PPG \\
\hline CEL & 0.14 & & & & & & & & & & & & & & & & & \\
\hline$P$ & 0.71 & & & & & & & & & & & & & & & & & \\
\hline $\mathrm{HE}$ & -0.14 & -0.11 & & & & & & & & & & & & & & & & \\
\hline$P$ & 0.72 & 0.77 & & & & & & & & & & & & & & & & \\
\hline SE & 0.14 & 0.11 & -1.00 & & & & & & & & & & & & & & & \\
\hline$P$ & 0.72 & 0.77 & 0.00 & & & & & & & & & & & & & & & \\
\hline ADF & 0.10 & 0.75 & 0.10 & -0.10 & & & & & & & & & & & & & & \\
\hline$P$ & 0.80 & 0.02 & 0.79 & 0.79 & & & & & & & & & & & & & & \\
\hline NDF & 0.49 & 0.48 & -0.34 & 0.34 & 0.10 & & & & & & & & & & & & & \\
\hline$P$ & 0.18 & 0.19 & 0.37 & 0.37 & 0.80 & & & & & & & & & & & & & \\
\hline HEM & 0.51 & 0.30 & -0.36 & 0.36 & 0.14 & 0.97 & & & & & & & & & & & & \\
\hline$P$ & 0.16 & 0.43 & 0.34 & 0.34 & 0.72 & 0.00 & & & & & & & & & & & & \\
\hline LIG & -0.24 & 0.14 & 0.33 & -0.33 & 0.55 & -0.34 & -0.46 & & & & & & & & & & & \\
\hline$P$ & 0.53 & 0.72 & 0.38 & 0.38 & 0.13 & 0.38 & 0.21 & & & & & & & & & & & \\
\hline DM & 0.14 & 0.15 & -0.65 & 0.65 & 0.02 & 0.07 & 0.07 & -0.21 & & & & & & & & & & \\
\hline$P$ & 0.71 & 0.70 & 0.06 & 0.06 & 0.95 & 0.85 & 0.87 & 0.59 & & & & & & & & & & \\
\hline TN & -0.54 & 0.32 & 0.32 & 0.32 & 0.74 & -0.21 & -0.38 & 0.66 & -0.23 & & & & & & & & & \\
\hline$P$ & 0.13 & 0.40 & 0.40 & 0.40 & 0.03 & 0.59 & 0.31 & 0.05 & 0.55 & & & & & & & & & \\
\hline TGW & 0.47 & -0.42 & -0.13 & 0.13 & -0.67 & 0.34 & 0.50 & -0.38 & 0.08 & -0.61 & & & & & & & & \\
\hline$P$ & 0.20 & 0.26 & 0.73 & 0.73 & 0.05 & 0.37 & 0.17 & 0.31 & 0.83 & 0.08 & & & & & & & & \\
\hline RSD & -0.54 & -0.13 & 0.69 & -0.69 & 0.13 & 0.29 & -0.32 & 0.36 & -0.26 & 0.26 & -0.32 & & & & & & & \\
\hline$P$ & 0.13 & 0.74 & 0.04 & 0.04 & 0.74 & 0.45 & 0.40 & 0.33 & 0.50 & 0.50 & 0.39 & & & & & & & \\
\hline SST & -0.02 & -0.54 & 0.58 & -0.58 & -0.05 & 0.16 & -0.15 & 0.70 & -0.52 & 0.18 & 0.12 & 0.54 & & & & & & \\
\hline$P$ & 0.97 & 0.13 & 0.11 & 0.11 & 0.91 & 0.68 & 0.70 & 0.04 & 0.15 & 0.65 & 0.76 & 0.14 & & & & & & \\
\hline IST & -0.15 & 0.03 & 0.73 & -0.73 & 0.13 & -0.08 & -0.11 & 0.19 & -0.41 & 0.04 & 0.22 & 0.90 & 0.49 & & & & & \\
\hline$P$ & 0.69 & 0.94 & 0.02 & 0.02 & 0.75 & 0.84 & 0.78 & 0.62 & 0.28 & 0.92 & 0.56 & 0.00 & 0.18 & & & & & \\
\hline TAN & 0.18 & -0.30 & -0.01 & 0.01 & -0.49 & -0.34 & -0.22 & -0.42 & -0.13 & -0.50 & -0.13 & -0.16 & -0.21 & -0.05 & & & & \\
\hline$P$ & 0.65 & 0.43 & 0.98 & 0.98 & 0.18 & 0.37 & 0.56 & 0.26 & 0.74 & 0.17 & 0.74 & 0.67 & 0.60 & 0.90 & & & & \\
\hline PS & 0.43 & -0.32 & -0.30 & 0.30 & -0.70 & 0.41 & 0.57 & -0.56 & 0.17 & -0.80 & 0.88 & -0.22 & -0.07 & -0.04 & -0.04 & & & \\
\hline$P$ & 0.25 & 0.40 & 0.43 & 0.43 & 0.04 & 0.27 & 0.11 & 0.11 & 0.67 & 0.01 & 0.00 & 0.56 & 0.85 & 0.92 & 0.93 & & & \\
\hline SA & -0.21 & -0.09 & 0.55 & -0.55 & 0.16 & 0.07 & 0.04 & 0.43 & -0.48 & 0.19 & -0.25 & 0.84 & 0.53 & 0.90 & -0.16 & -0.13 & & \\
\hline$P$ & 0.58 & 0.82 & 0.12 & 0.12 & 0.67 & 0.84 & 0.92 & 0.25 & 0.19 & 0.62 & 0.52 & 0.01 & 0.14 & 0.00 & 0.68 & 0.75 & & \\
\hline PPG & -0.19 & -0.05 & 0.49 & -0.49 & 0.17 & 0.10 & 0.06 & 0.39 & -0.47 & 0.15 & 0.25 & 0.81 & 0.95 & 0.85 & -0.21 & -0.08 & 0.99 & \\
\hline$P$ & 0.63 & 0.89 & 0.18 & $0 . .18$ & 0.66 & 0.80 & 0.88 & 0.30 & 0.20 & 0.70 & 0.51 & 0.01 & 0.06 & 0.00 & 0.59 & 0.84 & 0.00 & \\
\hline $\mathrm{RD}$ & 0.23 & 0.64 & -0.69 & 0.69 & 0.30 & 0.58 & 0.51 & -0.34 & 0.40 & -0.04 & 0.06 & -0.54 & -0.65 & -0.44 & -0.40 & 0.25 & -0.40 & -0.31 \\
\hline$P$ & 0.55 & 0.06 & 0.03 & 0.03 & 0.42 & 0.10 & 0.16 & 0.37 & 0.29 & 0.92 & 0.87 & 0.14 & 0.05 & 0.24 & 0.28 & 0.52 & 0.29 & 0.42 \\
\hline
\end{tabular}

Bold indicates a significant correlation $(P<0.05) ; P=$ significance value; $S T=$ starch; $C E L=$ cellulose, $H E=$ hard endosperm; $S E=$ soft endosperm; $A D F=$ acid detergent fiber; NDF= neutral detergent fiber; $\mathrm{HEM}=$ hemicellulose; $\mathrm{LIG}=$ lignin; $\mathrm{DM}=$ dry matter; $T \mathrm{~T}=$ total nitrogen; $\mathrm{TGW}=$ thousand-grain weight; RSD= predicted ruminal starch digestion; SST= soluble starch; IST= insoluble starch; TAN= tannins; PS= particle size (geometric mean diameter); $S A=$ surface area; PPG= particles per gram; $\mathrm{RD}=$ relative density. 
HÍBRIDOS DE SORGO Y SU RELACIÓN CON EL CONTENIDO DE ALMIDÓN Y DIGESTIÓN RUMINAL

medidas en los híbridos se muestran en el Cuadro 5. Las características que obtuvieron una mayor intensidad de correlación simple $(P<0.05)$ fueron, para las físicas: 1$)$ TP con $\mathrm{N}$ $(r=-0.80)$ y con FDA $(r=-0.70) ; 2)$ PGM con FDA $(r=-0.67)$ y con TP $(r=0.88) ; 3)$ PPG con DRUM ( $r=0.81)$; 4) DR con END-DU ( $r=$ $0.69)$ con END-BL $(r=-0.69)$ y con ALM-SOL $(r=0.70)$ y 5) SA con ALM-INSOL $(r=0.85)$ con ALM-SOL $(r=0.70)$ y con DRUM ( $r=$ 0.84 ), mientras que las relaciones para las características químicas fueron: 1) N con LIG $(r=0.66)$ y con FDA $(r=0.74) ; 2)$ FDN con HEMICEL $(r=0.97)$; 3) FDA con CEL $(r=$ $0.75)$; 4) LIG con ALM-SOL $(r=0.70)$. La relación de endospermos blando y duro con la fracción de almidón insoluble fue de 0.73 y -0.73, respectivamente. Se detectó una alta relación $(r=0.90)$ de la DRUM y ALM-INSOL, mientras que la relación de endospermos blando y duro con la DRUM fue de 0.69 .

Ecuaciones de predicción

Las gráficas de las relaciones detectadas en algunas características fisicoquímicas

Figura 1. Relación entre la densidad relativa (RD) y el \% de endospermo duro (HE)

Figure 1. Relationship between relative density (RD) and the percentage of hard endosperm (HE, \%)

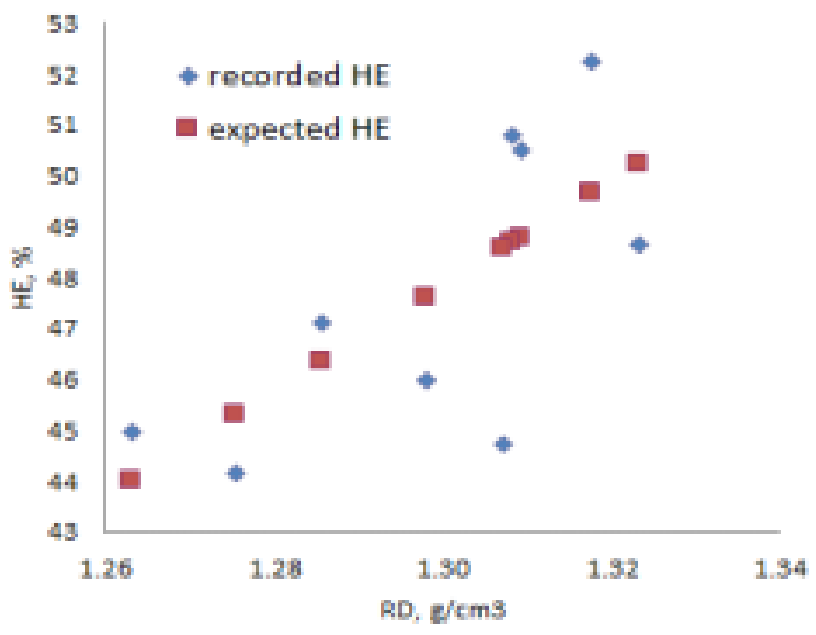

Expected HE= $-86.222+(103.111 * \mathrm{RD}),\left(P=0.04, \mathrm{r}^{2}=0.48\right)$. characteristics. Protein content increased as ADF content increased (expected $\mathrm{TN}=0.40+$ $\left[0.11 *\right.$ ADF]; $\left.P=0.03, \quad r^{2}=0.53\right)$. Relative density exhibited a positive correlation $(P<0.04)$ to HE content (Figure 1): grains with RD $>1.28$ accounted for $45 \%$ of $\mathrm{HE}$ content, but those with $\mathrm{RD}>1.32$ accounted for $50 \%(\mathrm{HE}, \%=$ $\left.-86.222+103.111 \mathrm{RD} ; r^{2}=0.48\right)$.

A positive correlation was present between RSD and calculated SA such that for every $\mathrm{cm}^{2}$ increase in SA, RSD increased 0.35 percentage points (RSD, $\%=59.93-0.35 S A ; r^{2}=0.70$, $P<0.01$ ) (Figure 2). In contrast, estimated RSD decreased as the grain $\mathrm{HE}$ proportion increased $\left(\mathrm{RSD}, \%=79.63-(0.285 \mathrm{HE}) ; r^{2}=\right.$ $0.47 ; P=0.04$ ) (Figure 3). Percentages for RSD surpassed $67 \%$ when HE dropped below $45 \%$, and decreased $0.28 \%$ for each percentage point increase in HE above $45 \%$. Based on the significant relationships identified between RSD and HE, SA and RD, a multiple regression equation was developed to predict RSD. Inclusion of these three variables raised the RSD determination coefficient by $77 \%$ :

Figura 2. Relación entre la digestibilidad ruminal (RSD) y la superficie de área (SA)

Figure 2. Relationship between predicted ruminal starch digestion (RSD) and surface area (SA)

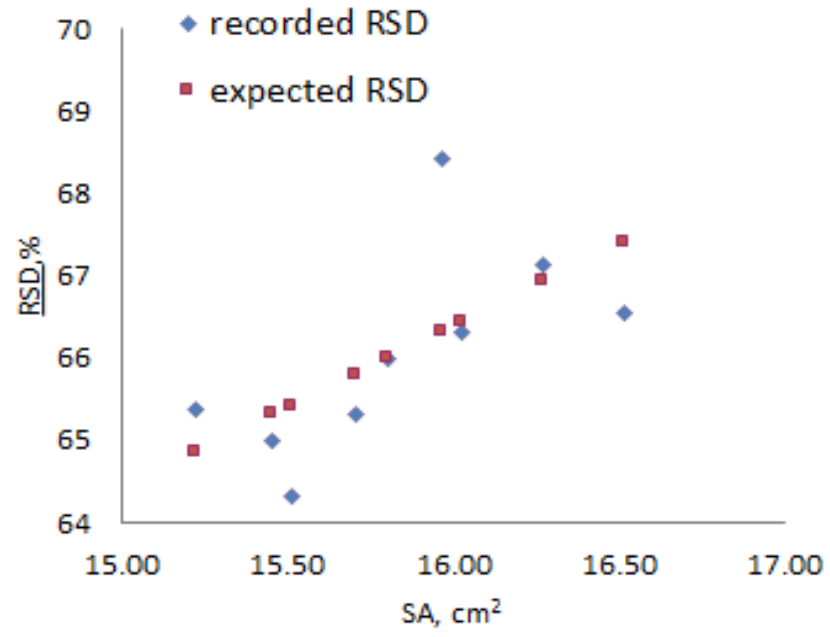

Expected RSD $=59.93-(0.35 * \mathrm{SA}) .\left(P=0.005, \mathrm{r}^{2}=0.70\right)$. 
estadísticamente significativas, se muestran en las Figuras 1 a 3. El contenido de proteína del grano se incrementó a medida que el contenido de la FDA aumentó, NT esperado $=0.40+$ (0.11* FDA). $\left(P=0.03, r^{2}=0.53\right)$. La densidad relativa (DR) resultó en una correlación positiva $(P<0.04)$ con el contenido de endospermo duro (Figura 1), granos con densidades relativas $>1.28$ representan un $45 \%$ de contenido de END-DU los cuales aumentan a $50 \%$ de ENDDU con DR de 1.32 (END-DU, $\%=-86.222+$ 103.111DR; $\left.r^{2}=0.48\right)$. Se observó una correlación positiva entre la DRUM y la superficie de área calculada (Figura 2). Por cada $\mathrm{cm}^{2}$ que aumente la SA la DRS aumentará en 0.35 unidades porcentuales (DRUM, \% $=59.93$ $\left.0.35 \mathrm{SA} ; r^{2}=0.70, P<0.01\right)$.

La DRUM disminuyó a medida que se incrementó la proporción de END-DU en el grano (Figura 3). Digestibilidad ruminal, $\%=79.63-(0.285 E N D-$ DU); $r^{2}=0.47(P=0.04)$. La digestibilidad ruminal del almidón excede el 67 \% cuando la proporción de endospermo duro fue menor a $45 \%$ disminuyendo en $0.28 \%$ por cada unidad porcentual de incremento de proporción de endospermo duro por encima de $45 \%$. Como resultado de las relaciones significativas detectadas entre el contenido de END-DU, de la SA y la DR con DRUM se desarrolló una ecuación de regresión múltiple para la predicción de la DRUM. La inclusión de las tres variables permite incrementar $77 \%$ el coeficiente de determinación para DRUM: DRUM, $\%=74.058$ - 0.108END-DU + 0.027SA - 5.887DR, $P<0.05$.

\section{DISCUSIÓN}

Características físicas

El promedio de MS registrado en el presente estudio es $6.7 \%$ superior al informado $(86.67 \%$ ) por algunos investigadores(23) y $2.7 \%$ superior al valor tabulado por Drinah et al(24) y por NRC(25). La variación en el contenido de MS se debe principalmente a cambios por las condiciones de almacenamiento previos al análisis. McCollough et a/(26) encontraron una rango de 87.7 a $91.4 \%$ de MS en siete
Figura 3. Relación entre el porcentaje de endospermo duro (HE) y la digestibilidad ruminal (RSD)

Figure 3. Relationship between the percentage of hard endosperm (HE) and predicted ruminal starch digestion (RSD)

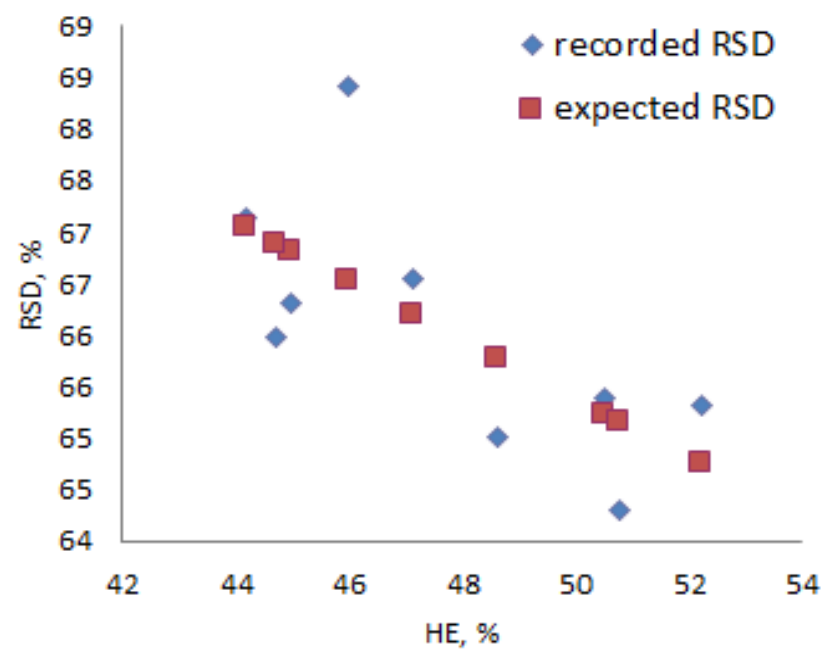

Expected RSD $=79.63-(0.285 * \mathrm{HE})\left(P=0.04, \mathrm{r}^{2}=0.47\right)$.

$\mathrm{RSD}, \%=74.058-0.108 \mathrm{HE}+0.027 \mathrm{SA}-$ 5.887RD $(P<0.05)$.

\section{DISCUSSION}

Physical characteristics

Average DM in the present study was $6.7 \%$ higher than the $86.67 \%$ reported in previous studies(23), and $2.7 \%$ more than reported by Drinah et a/(24) and NRC(25). Variation in DM content is mainly due to changes in pre-analysis storage conditions; for instance, DM ranges of 87.7 to $91.4 \%$ have been reported in seven hybrids(26). Another possible contributor to DM content variation is the HE:SE proportion in each hybrid. During grain development, water is largely stored in the SE due to its loose structure(1). This would explain the trend $(P=0.06)$ towards a negative $\mathrm{SE}$ to $\mathrm{DM}$ ratio $(r=-0.65)$ in the present study.

Sorghum grain diameter varies from 3 to $8 \mathrm{~mm}$, depending on variety(27). With a $3.168 \pm 0.005$ $\mathrm{mm}$ average $\mathrm{PS}$, the hybrids studied here can 
híbridos evaluados. Otra posible causa que contribuye a la variación en el contenido de la MS es la proporción de END-DU:END-BL que presente cada híbrido. Durante el desarrollo del grano, el agua se moviliza y almacena mayormente en el END-BL debido a su estructura más laxa(1). Lo anterior puede explicar la tendencia $(P=0.06)$ a la relación negativa ( $r=-0.65)$ observada para la proporción de END-BL con MS ( $r=-0.65)$ encontrada en este estudio. Dependiendo de la variedad, el diámetro del grano de sorgo oscila de 3 a $8 \mathrm{~mm}$ (27); por lo anterior, los híbridos utilizados en este trabajo son de calibre pequeño, ya que el valor TP promedio obtenido fue de $3.168 \pm 0.005 \mathrm{~mm}$. La prueba de TP es una herramienta útil para conocer el diámetro y grado de molienda en las partículas de harina o del grano rolado(28). Para el grano entero, es mucho menos frecuente el uso de esta prueba, por esto, los datos presentados en este trabajo pueden ser muy útiles como una nueva referencia sobre esta determinación. El TP tuvo correlaciones significativas $(P<0.04)$ con el contenido de $\mathrm{N}$, con la FAD y con el PMG, lo que indica que la determinación del TP en grano entero puede utilizarse como un elemento adicional para valorar al grano de sorgo. Adicionalmente, el cálculo de la SA, cálculo derivado del TP, demostró ser útil para la predicción de la DRUM $(r=0.84)$. El valor promedio observado para PMG fue $21.1 \%$ menor al valor promedio informado en otro trabajo(29) pero similar a los valores de 20 a $30 \mathrm{~g}$ reportados por Magness et al(30). Estos investigadores determinaron PMG pesando 100 granos y multiplicando el resultado por 10. En cambio, tal como lo marca la técnica del Rice Knowledge Bank(31), para el presente estudio se contaron y pesaron 1,000 granos al azar. Físicamente, la medición del PMG es una medida relativa del tamaño del grano, como resultado fue la alta correlación determinada en el presente trabajo entre TP y el PMG $(r=0.88)$ por lo que la ecuación de regresión: TP, $\mu \mathrm{m}=2438.07$ + (29.65PMG) permite establecer los valores de TP promedio, y puede servir de referencia be considered to have small grains. Particle size (PS) is a useful tool for understanding diameter and degree of grind in meal particles or rolled grain(28). Rarely has this test been applied to whole grains, meaning the present data could be used to create a new reference for sorghum whole grain PS. Significant correlations $(P<0.04)$ were observed between PS and TN, ADF and TGW; in whole sorghum grain, this parameter can therefore be used as an additional evaluation element. In addition, calculation of SA, which is derived from PS, was shown to be useful in predicting RSD $(r=0.84)$.

In the present study, average TGW was $21.1 \%$ less than reported in one previous study(29), but similar to the 20 to $30 \mathrm{~g}$ reported in another(30). Discrepancies between studies may be due to slight differences in technique; for instance, in the above studies 100 grains were weighed out and this figure multiplied by ten, but in the present study 1,000 grains were randomly counted out and weighed(31). Thousand-grain weight (TGW) is a relative measure of grain size, which is why the correlation between PS and TGW was high $(r=0.88)$ in the present results. Moreover, for this reason the regression equation (PS, $\mu \mathrm{m}=$ $2438.07+[29.65 T G W]$ ) allows for calculation of average PS, and can function as a reference in calculating whole grain sorghum size and weight.

Average RD $\left(1.299 \mathrm{~g} / \mathrm{cm}^{3} \pm 0.02\right)$ in the present study was 6.2 to $3.7 \%$ lower than reported in some previous studies $(29,32)$. It did fall within the 1.14 to $1.37 \mathrm{~g} / \mathrm{cm}^{3}$ range reported by Chandrashekar and Kirleis(33), although they used dehulled sorghum grain and did not describe the technique used to measure RD. Differences in RD measurement techniques and the substances used in them (e.g. nitrogen, xylene, distilled water) make valid comparisons difficult with other published data. Use of distilled water tends to underestimate RD because the degree of grain humidification depends on liquid surface tension and other reactions that can occur during contact between the grain and 
para determinaciones del tamaño y peso del grano entero de sorgo.

El promedio de DR $\left(1.299 \mathrm{~g} / \mathrm{cm}^{3} \pm 0.02\right)$ observado en el presente estudio es 6.2 y $3.7 \%$ inferior a los informados en otras investigaciones $(29,32)$, aunque coinciden con el rango de 1.14 a $1.37 \mathrm{~g} / \mathrm{cm}^{3}$ indicado por Chandrashekar y Kirleis(33); sin embargo, estos autores utilizaron sorgo descascarillado y no mencionan la técnica que usaron para medir la densidad relativa. Las diferencias entre las técnicas empleadas para medir DR, así como las diferencias entre las sustancias utilizadas (nitrógeno, xileno, agua destilada), hacen difícil establecer una comparación válida con el resto de los valores publicados. Se puede suponer que la determinación de la DR con agua destilada tiende a subestimar el valor de densidad, debido a que la humectación del grano depende de la tensión superficial del líquido y de otras interacciones que pudieran ocurrir durante el contacto del grano con el líquido, como es la formación de burbujas o la disolución de algún componente de la cascarilla que no fueron medidos ni controlados. La densidad es útil para determinar la proporción de END-DU en el grano de sorgo, y aunque los valores de correlación encontrados aquí son inferiores a los informados por otros(34), tuvieron un valor consistente de correlación que permitió establecer una ecuación de predicción de END-DU. Contrario a lo informado(29), no se detectó correlación entre la DR y el contenido de N. La concentración de $\mathrm{N}$ en grano de sorgo publicada, se encuentra en el rango de 1.15 a $2.64 \%(25,33,35)$.

En concordancia con lo expresado por Buffo et al(29), se observó una tendencia $(P=0.06)$ de correlación negativa $(r=-0.54)$ entre el porcentaje de almidón y el contenido de N, ya que un menor porcentaje de proteína puede ser resultado de mayores condiciones favorables en el cultivo que permitan un mayor almacenamiento de almidón en el grano durante su desarrollo. the liquid; examples include bubble formation and dissolution of a component in the hull that was not measured or controlled. Relative density (RD) is useful in determining the $\mathrm{HE}$ proportion in a sorghum grain, although the correlation values observed here are lower than reported elsewhere(34). However, the value in the present data was consistent, allowing use of a predictive equation for $\mathrm{HE}$. In contrast to previous studies(29), no correlation between RD and TN content was observed here. Published sorghum grain $\mathrm{N}$ contents range from 1.15 to $2.64 \%(25,33,35)$.

The tendency $(P=0.06)$ towards a negative correlation $(r=-0.54)$ between ST percentage and TN content coincides with Buffo et al(29). A lower protein percentage can be the result of more favorable cultivation conditions, which allow greater starch storage in the grain during development.

Quantification of fibrous fraction components (NDF, ADF, hemicellulose, cellulose and lignin) is uncommon in cereals because their fiber content is quite low. For instance, when sorghum grain is used as an energy source in ruminant feeds, fiber requirements must be covered by other dietary components. The present values for fiber components are very similar to those reported elsewhere $(25,36)$. Most $(82.3 \%)$ of the fiber fraction is represented by the pericarp, most of which is considered insoluble. Lignin accounts for $20 \%$ of the cell wall in sorghum grain(27), suggesting that evaluation of the fiber fraction could help in determining the amount of pericarp in this organ. Neutral detergent fiber (NDF) and ADF are measurements done by chemical and physical methods, and HEMI and CEL are estimations derived from these two measurements. High correlations between them can therefore to be expected; in fact, the correlation was $r=0.97$ between NDF and HEMI, and $r=0.75$ between ADF and CEL. The high correlations between $\mathrm{TN}$ and $\mathrm{ADF}$, and $\mathrm{TN}$ and LIG were unexpected since $80 \%$ of grain $\mathrm{N}$ content is in the endosperm and only $4 \%$ is in the fibrous structure(37). 
La determinación de los componentes de la fracción fibrosa (FDN, FDA, hemicelulosa, celulosa y lignina) no es muy común en los cereales debido a que su contenido de fibra es muy bajo, y a que el grano de sorgo que es utilizado en las dietas de rumiantes es como fuente de energía, y el aporte de fibra es cubierto por el resto de los componentes de la misma. Aún así, los valores determinados en el presente estudio son muy similares a los valores informados previamente $(25,36)$. La mayor parte de la fracción fibra se encuentra en el pericarpio $(82.3 \%$ ) y la mayoría de ésta puede considerarse fibra insoluble. Considerando que en el grano de sorgo la lignina constituye el $20 \%$ de la pared celular(27); entonces, la evaluación de las fracciones de la fibra podría ser útil para determinar la cantidad de pericarpio presente en el grano. En virtud de que FDN y FDA son determinaciones (a través de procesos químicos y físicos) y HEMICEL y CEL son estimaciones derivadas de ambas determinaciones; es de esperarse correlaciones altas entre ellas mismas que fueron observadas tanto para FDN y HEMICEL $(r=0.97)$ como para FDA y CEL $(r=0.75)$. La relación detectada para FDA $(r=0.74, P=0.04)$ y para LIG $(r=$ $0.66)$ con el contenido total de $\mathrm{N}$ es sorpresiva, ya que el $80 \%$ del total del nitrógeno (PC) del grano se encuentra en el endospermo y sólo el $4 \%$ en las estructuras fibrosas(37).

El sorgo varía ampliamente en la composición y contenido de compuestos fenólicos. Basados en el contenido de taninos extraíbles el grano de sorgo se clasifica, de menor a mayor contenido de taninos, como tipo I, II y III(15). En los últimos años se han dirigido los esfuerzos a la producción de variedades e híbridos libres de taninos, ya que estos reducen la disponibilidad o digestibilidad de los nutrimentos del sorgo reduciendo su valor alimenticio; de hecho, en la actualidad el $99 \%$ de sorgos producidos en los Estados Unidos son libres de taninos(38). Por lo anterior, no es sorpresivo que las variedades aquí estudiadas resultaran prácticamente libres de estos compuestos.
Sorghum varies widely in phenolic compounds content and composition. Based on extractable tannins content (lesser to greater), sorghum grain is classified as type I, II or III(15). Recently developed sorghum varieties and hybrids are tannin-free because this compound reduces nutrient availability or digestibility, thereby reducing dietary value. In the United States, $99 \%$ of the sorghum produced currently is tannin-free(38). It is therefore no surprise that all the studied hybrids had essentially no detectable tannins.

In the nine analyzed sorghum hybrids, average starch (ST) content was $70 \%(1)$, which is within the 61.9 to $83 \%$ range reported previously $(39,40)$. Variation in starch content is largely attributable to differences between hybrids, fertilization practices and quantification techniques. Starch content in the present study did not significantly correlate to any of the other measured characteristics, which coincided with a previous study(41). This prevents any precise, indirect estimation of starch content via these other compounds. In vivo ruminal starch digestion of ground sorghum starch is approximately $65.9 \%(40,42,43)$. In two reviews covering over eighty studies that used more than $65 \%$ sorghum starch in the diet, in vivo starch ruminal digestibility averaged $66.87 \%(44,45)$. In another study, ruminal digestibility of ground sorghum was $60 \pm$ $12 \%(46)$, and was found to vary depending in genotype, hybrid and cultivation conditions. The average (RSD) estimated in the present study $(66.04 \pm 1.20 \%)$ was very similar to these figures. This parameter was estimated based on the SST and IST fractions in the nine studied hybrids(17). Considering that the coefficient is $74 \%$ in this technique, the in vivo ruminal digestion estimated here is near expected levels for the studied hybrids.

Quantitative measurement of sorghum grain $\mathrm{HE}$ and SE using the technique of Kirleis et a/(18) is effective, but requires extensive experience, precision and manual dexterity. The modifications made to this technique in the present study 
El contenido de almidón en el sorgo promedia $70 \%(1)$, aunque está en el rango de 61.9 a 83 $\%(39,40)$, esta variación es principalmente atribuible a la diferencia genética entre híbridos, a las prácticas de fertilización y a las técnicas utilizadas en la determinación de almidón en el grano. En concordancia con lo publicado(41), el contenido de ALM no presentó ninguna correlación significativa con el resto de las características medidas, por tanto, no es posible estimar con precisión la cantidad de almidón contenido en el grano por medio de determinaciones indirectas a través del resto de los compuestos. Reportes previos indican que la digestibilidad ruminal del almidón de sorgo molido in vivo es aproximadamente del $65.9 \%(40,42,43)$. En revisiones realizadas donde se incluyen más de 80 experimentos y se utilizó más del $65 \%$ de sorgo molido en la dieta, la digestibilidad ruminal del almidón in vivo promedia $66.87 \%(44,45)$. Ambruster $(46)$ indica que la digestiblidad ruminal del sorgo molido es de $60 \pm 12 \%$, y que esta variación obedece al genotipo, al tipo de híbrido y a las condiciones agronómicas donde se desarrolló el grano. Todo lo anterior concuerda estrechamente con el valor promedio estimado de digestibilidad ruminal (66.04 $\pm 1.20 \%$ ) obtenido en el presente estudio utilizando la técnica de Rodríguez et a/(17) a través de la determinación de las fracciones soluble e insoluble de almidón. Considerando que el coeficiente de determinación de la técnica de Rodríguez es de $74 \%$, entonces, la estimación de la digestibilidad ruminal obtenida en la presente investigación es muy cercana a lo esperado in vivo para los híbridos estudiados.

Aunque la técnica de Kirleis et al(18) es muy útil para medir cuantitativamente las áreas de END-DU y END-BL de grano de sorgo, su uso tiene varias limitantes, ya que se requiere de mucha experiencia, precisión y habilidad manual. La modificaciones realizadas al utilizar el corte con bisturí para la exposición del centro del germen y endospermo, al uso de microfotografías digitales y la medición de áreas a través del software Adobe Photoshop Elements hace a make it much more practical and rapid since the digital images can be modified at any time, any modifications can be reversed, marking of the area to be measured does not require a continuous movement, and the equipment and software are widely used and reasonably priced.

A sorghum grain consists of three morphological parts: pericarp, germ, and endosperm. The proportions of basic starch structures (amylose and amylopectin) in the hard (HE) and soft endosperm (SE) affect starch digestibility(47). Depending on genotype, HE proportions can vary from 30 to $80 \%$ of the total endosperm. Based on Waterman et a/(48), grains are classified into three classes of hardness: hard $(\sim 80 \%)$, intermediate $(\sim 50 \%)$, and soft $(\sim 30 \%)$. The nine studied hybrids had an average HE:SE ratio of $47: 53$, classifying them as of intermediate hardness; only four of the nine exhibited differences $(P<0.05)$. In corn grain, the HE:SE ratio has been proposed as one of the most important parameters limiting starch digestion in ruminants(10). In an in vivo digestion study using different corn hybrids, a high correlation was observed between the proportion of $\mathrm{HE}$ and predicted ruminal starch digestion (RSD): RSD = 78.91 -18.13 HE; $\mathrm{R}^{2}=$ 0.90 . For sorghum, very little data is available on the effect of endosperm proportions in ruminal starch digestion(11).

Starch molecular constitution in sorghum clearly affects starch digestibility, but so do the type of starch granule encapsulation and the percentage and type of sorghum proteins (kafirins). Accurate prediction of starch digestibility in sorghum apparently requires use of more factors. For instance, when the predictive equation was modified to include SA correlated significantly to IST $(r=0.85)$ and SST $(r=0.70)$, and RD significantly correlated to HE $(r=0.69)$ and SE $(r=-0.69)$, its determination coefficient increased to $77 \%$ : RSD, $\%=74.058-0.108 \mathrm{HE}+$ $0.027 \mathrm{SA}$ - 5.887RD. 
esta prueba más práctica y rápida, debido a que las fotografías pueden ser modificadas en cualquier momento, se pueden revertir las modificaciones hechas a las mismas, la delimitación de las áreas no requiere de un trazo o movimiento de la mano continuo a través de todo el perímetro del área a medir, y los equipos y software utilizado no son de alto costo y son de uso común.

El grano de sorgo está compuesto por tres partes morfológicas: pericarpio, germen y endospermo. La proporción de las estructuras básicas del almidón (amilosa y amilopectina) y del endospermo vítreo o duro (END-DU) y blando o harinoso (END-BL) afectan la digestibilidad del almidón(47). Dependiendo del genotipo, la proporción de END-DU va desde un 30 a un $80 \%$ del total del endospermo, y la clasificación de dureza de acuerdo a Watterson et al(48) es: duro ( $80 \%)$, intermedio $(\sim 50 \%)$ y blando $(\sim 30 \%)$. Por lo anterior, de acuerdo a la proporción de END-DU: ENDBL de 47:53, los híbridos aquí estudiados se pueden considerar como "intermedios", y sólo 4 de los 9 híbridos mostraron diferencia $(P<0.05)$. Recientemente se ha sugerido que la proporción de endospermo duro:blando es uno de los factores más importantes que limita la digestión del almidón en el grano de maíz en rumiantes(10). En un experimento de digestión in vivo en el cual utilizaron distintos híbridos de maíz(49), detectaron una alta correlación entre la proporción de endospermo duro y la digestibilidad del almidón (DRUM): DRUM= 78.91 -18.13 END-DU; $R^{2}=0.90$. Sin embargo existe información limitada sobre las relaciones de la proporción de endospermos con la digestibilidad ruminal del almidón en el sorgo(11). No sólo la constitución molecular del almidón por sí misma afecta la digestibilidad del almidón, también el tipo de encapsulamiento de gránulos de almidón y el porcentaje y tipo de proteínas del sorgo (kafirinas) presentes influyen en esta relación. Aparentemente, para el sorgo deben considerarse el incluir más factores para incrementar la fuerza de predicción. El incluir a la superficie de área (SA) relacionada

\section{CONCLUSIONS AND IMPLICATIONS}

All the evaluated physicochemical variables for grains from the nine sorghum hybrids were within previously published values. No significant relationship was identified between the physical or chemical variables and total grain starch content. Indirect quantification of starch content using other compounds in the grain is therefore not possible. Unlike in corn, the hard endosperm proportion in sorghum grain is not a good indicator for measuring ruminal starch digestion from the soluble and insoluble starch fractions. However, adding relative density and surface area to the equation significantly raises its predictive power.

\section{ACKNOWLEDGEMENTS}

Financial support for the research reported here was provided by the Dirección General de Asuntos del Personal Académico, UNAM (Proj. No. PAPIIT IN206006).

End of english version

significativamente con ALM-INSOL $(r=0.85)$ con ALM-SOL $(r=0.70)$ y a la densidad relativa (DR) relacionada significativamente con ENDDU $(r=0.69)$ con END-BL $(r=-0.69)$ en la ecuación se incrementó el coeficiente de determinación a $77 \%$ : DRUM, \% $=74.058$ 0.108END-DU + 0.027SA - 5.887DR.

\section{CONCLUSIONES E IMPLICACIONES}

Las variables fisicoquímicas determinadas para las nueve variedades de sorgo se encuentran dentro de los rangos especificados en otras fuentes de información. De acuerdo a sus características principales, las variedades se consideran de dureza intermedia y libre de taninos. No se detectó ninguna relación significativa entre las variables físicas o químicas 
con el contenido total de almidón en el grano, por tanto, no es posible estimar con precisión la cantidad de almidón contenido en el grano por medio de determinaciones indirectas a través del resto de los compuestos. Contrario al maíz, la proporción de endospermo duro en el grano de sorgo no es un buen indicador para determinar la digestibilidad ruminal del almidón a través de las fracciones de almidón soluble e insoluble; sin embargo, cuando se añaden la densidad relativa y superficie de área a la ecuación, ésta incrementa su poder de predicción en forma significativa.

\section{AGRADECIMIENTOS}

Se agradece el apoyo financiero para la realización del trabajo a través del proyecto PAPIIT IN206006 de la Dirección General de Asuntos del Personal Académico de la UNAM.

\section{LITERATURA CITADA}

1. Huntington GB. Starch utilization by ruminants: From basics to the bunk. J Anim Sci 1997;(75):852-67.

2. Duodu KG, Taylor JRN, Belton PS, Hamaker BR. Factors affecting sorghum protein digestibility. J Cereal Sci 2003; (38): 117-131.

3. Kumar AA, Reddy BV, Sharma HC, Hash CH, Rao PS, Ramaiah B, Reddy PS. Recent advances in sorghum genetic enhancement research at ICRISAT. Am J Plant Sci 2011;(2):589-600.

4. Fernholz M. Evaluation of four sorghum hybrids through the development of sorghum flour tortillas. [MSc Thesis]. Manhattan Kansas, USA: Kansas State University; 2008.

5. Streeter MN, Wagner DG, Hibberd CA, Owens FN. Comparison of corn with four sorghum grain hybrids: Site and extent of digestion in steers. J Anim Sci 1990; (68):3429.

6. Streeter MN, Wagner DG, Owens FN, Hibberd CA. The effect of pure and partial yellow endosperm sorghum grain hybrids on site and extent of digestion in beef steers. J Anim Sci 1991;(69):2571.

7. Stock RA. Nutritional benefits of specialty grain hybrids in beef feedlot diets. J Anim Sci 1999;(82):208-212.

8. Hicks C, Bean SR, Lookhart GL, Pedersen JF, Kofoid KD, Tuinstra MR. Genetic analysis of kafirins and their phenotypic correlations with feed quality traits, in vitro digestibility and seed weight in grain sorghum. Cereal Chem 2001;(78):412-416.
9. Tesso T, Ejeta G, Chandrashekar A, Huang CP, Tandjung A, Lewamy M, Axtell JD, Hammaker BR. A novel modified endosperm texture in a mutant high-protein digestibility/ high-lysine grain sorghum (Sorghum bicolor (L.) Moench). Cereal Chem 2006;(83):194-201.

10. Owens FN, Soderlund S. Corn grain: Assessing nutritional value for livestock. Minnesota Cattle Feeders Day Publication. Univ Minnesota. BP-1004. http:// www.mnbeef.umn.edu/cattle-feeder/2010/BP1004Owens.pdf. Accessed Aug 8, 2014.

11. Montiel MD, Elizalde JC, Santini F, Giorda L. Características físicas y químicas del grano de sorgo. Relación con la degradación ruminal en bovinos. Arch Zootec 2011; (60):533-541.

12. AOAC. Official methods of analysis. $15^{\text {th }}$ ed. Arlington, VA, USA: Association of Official Analytical Chemists; 1990.

13. Baker S, Herrman T. Evaluating particle size. Feed Manufacturing. Kansas State Univ. Agric Exp Sta. Coop Ext Service. 2002. http://www.ksre.ksu.edu/bookstore/ pubs/mf2051.pdf Accessed Aug 8, 2014.

14. Van Soest PJ, Robertson JB, Lewis BA. Methods for dietary fiber, neutral fiber and non starch polysaccharides in relation to animal nutrition. J Dairy Sci 1991;74:35883597.

15. Price ML, Van Scoyoc S, Butler LA. Critical evaluation of the vanillin for tannin in sorghum grain. J Agric Food Chem 1978; (26): 1214-1218.

16. Zinn RA. Influence of flake density on the comparative feeding value of steam-flaked corn for feedlot cattle. J Anim Sci 1990;(68):767-775.

17. Rodríguez S, Calderón JF, Zinn RA. Variation in ruminal starch digestion due to dry rolling versus steam flaking corn and sorghum can be reliably predicted based on changes in starch solubility and $6-\mathrm{H}$ amylase reactive insoluble starch. Proc. Western Sect. Am Soc Anim Sci 2001;(52): 529-530.

18. Kirleis AW, Crosby KD, Housley TL. A method for quantitatively measuring vitreous endosperm area in sectioned sorghum grain. Cereal Chem 1984;(61):556558.

19. Knoll T, Hamburg M, Pauliger M, Rys $C$, Schaefer $S$, Hildt M. Adobe Photoshop Elements. Adobe Systems Incorporated. (c) 1989-2001. U.S, patents pending.

20. SAS. SAS/STAT User's guide. [Computer program] 4th ed. Version 6. Cary NC, USA: SAS Institute Inc. 1990.

21. Cochran WG, Cox GM. Experimental designs. 2nd ed. Wiley Classics Library. New York, USA: John Wiley \& Sons; 1992.

22. Statistix for Windows. Versión 8.0, Tallahassee, (Florida) USA. Analytical software [on CD].

23. Yang $P$, Seib PA. Low-input wet-milling of grain sorghum for readily accessible starch and animal feed. Cereal Chem 1995; (72):498-503.

24. Drinah BG, Banda-Nyirenda, Vohra $\mathrm{P}$, Ingebretson KH. Nutritional evaluation of some varieties of sorghums (Sorghum bicolor L. Moench). Cereal Chem 1987;(64):413417.

25. NRC. Nutrient requirements of beef cattle (updated $7^{\text {th }}$ Rev. Ed.) Washington DC, USA: Natl Acad Press; 2000. 
26. McCollough RL, Riley JG, Drake CL, Roth GM. Feedlot performance on nine hybrid sorghum grains fed to steers winter, 1971-1972. Kansas Agric Exp Stat. Bull 557. 1972.

27. Dicko MH, Gruppen H, Traoré AS, Voragen AGJ, Berkel van WJH. Review. Sorghum grain as human food in Africa: Relevance of content of starch and amylase activities. Afr J Biotech 2006;(5):384-395.

28. Hales KE, Cole NA, Galyean ML, Leytem AB. Nutrient concentrations and proportions in particle size fractions of corn steam flaked to different bulk densities. Prof Anim Sci 2010; (26):511-519.

29. Buffo RA, Weller CL, Parkhurst AM. Relationships among grain sorghum quality factors. Cereal Chem 1998; (75):100104

30. Magness JR, Markle GM, Compton CC. Food and feed crops of the United States. [Interregional Research Project IR-4.] IR Bul 1, New Jersey Agric Exp Sta. New Brunswick. 1971.

31. Rice knowledge bank [Editorial]. 1000 Grain Weight. International Rice Research Institute.: http:// www.knowledgebank.irri.org/training/fact-sheets/ postharvest-management/rice-quality-fact-sheet-category/ item/measuring-white-rice-quality-fact-sheet. Accessed Aug 8, 2014

32. McCollough RL, Riley JG, Crake CL, Brent BE. Digestibility of seven hybrid grain sorghums and two hybrid corns [abstract]. J Anim Sci 1972;(35):270.

33. Chandrashekar A, Kirleis AW. Influence of protein on starch gelatinization in Sorghum. Cereal Chem 1988;(65):457462.

34. Maxson ED, Fryar WB, Rooney LW, Krishnaprased MN. Milling properties of sorghum grain with different proportions of corneous to floury endosperm. Cereal Chem $1971 ;(48): 478-490$

35. Mossé J, Huet JC, Baudet J. The amino acid composition of whole sorghum grain in relation to its nitrogen content. Cereal Chem 1988;(65):1988.

36. Ramírez E, Anaya AM, Mariscal G. Predicción de la composición química del grano de sorgo mediante espectroscopía de reflectancia cercana al infrarrojo (NIRS). Tec Pecu Mex 2005;(43):1-11.

37. Lochte-Watson, Weller CL, Eskridge KM. Fractional composition of grain sorghum (Sorghum bicolor) after wetpeeling in a centrifugal pump. Biol Sys Engin: Papers and
Publications. 2000. Paper 86. http://digitalcommons.unl.edu/ biosysengfacpub/86. Accessed Aug 8, 2014.

38. Awika, JM Rooney LW. Sorghum phytochemicals and their potential impact on human health. Phytochem 2004; (65): 1199-1221.

39. Hibberd CA, Wagner DG, Schemm RL, Mitchell Jr ED, Weibel DE, Hintz RL. Digestibility characteristics of isolated starch from sorghum and corn grain. J Anim Sci 1982; (55): 1490-1497.

40. Souilah R, Djabali D, Belhadi B, Mokrane $H$, Boudries $N$, Nadjemi B. In vitro starch digestion in sorghum flour from Algerian cultivars. Food Sci 2014;(3):251-259.

41. Hibberd CA, Wagner DG, Schemm RL, Mitchell J $r$ ED, Hintz $\mathrm{RL}$, Weibel DE. Nutritive characteristics of different varieties of sorghum and corn grain. J Anim Sci 1982;(55):665-672.

42. Hinman DD, Johnson RR. Influence of processing methods on digestion of sorghum starch in high concentrate beef cattle rations. J Anim Sci 1974;(39):417-422.

43. Hinman DD, Johnson RR. Influence of degree of micronization on the site and extent of sorghum starch digestion in beef cattle fed high concentrate rations. J Anim Sci 1974;(39):958-963.

44. Salinas J, Álvarez EG, Zinn RA. Influence of tempering on the feeding value of steam-flaked sorghum for feedlot cattle. Proc Western Sect. Am Soc Anim Sci 1999;(50):325330.

45. Owens FN, Zinn RA. Corn grain for cattle: Influence of processing on site and extent of digestion. Proc 19th Annual Southwest Nut \& Management Conf. Phoenix AZ. 2005;86-112.

46. Ambruster S. Steam-flaking grain for feedlot cattle: A consultant's perspective. Proc Cattle Grain Proces Symp. Oklahoma State Beef Ext. 2006. http://beefextension.com/ proceedings/cattle_grains06/06-7.pdf. Accessed Aug 8, 2014.

47. Hoseney RC, Davis AB, Herbers LH. Pericarp and endosperm structure of sorghum grain shown by scanning electron microscopy. Cereal Chem 1974;(51):552-558.

48. Watterson JJ, Shull JM, Kirleis AW. Quantitation of $\alpha-, \beta$ and $\gamma$ - kafirins in vitreous and opaque endosperm of sorghum bicolor. Cereal Chem 1993;(70):452-457.

49. Corona L, Owens FN, Zinn RA. Impact of corn vitreousness and processing of site and extent of digestion by feedlot cattle. J Anim Sci 2006;(84):3020-3031. 
